\title{
Uma realidade aumentada do IPB para a cidade de Bragança
}

\author{
Vitor Gonçalves a , Eduardo Elias Pereira da Silveira Filho ${ }^{\text {b }}$
}

${ }^{a}$ CIEB, Instituto Politécnico de Bragança, Bragança, Portugal, vg@ipb.pt, ${ }^{\text {b}}$ Universidade Federal de Goiás, Jataí, Brasil, eduardoepsf@outlook.com.

\section{Resumo}

No âmbito do Estágio de Iniciação Científica proporcionado pela plataforma de empreendedorismo, empregabilidade e inovação formativa do Instituto Politécnico de Bragança (IPB), desenvolveu-se o projeto "IPB: uma realidade aumentada" que pretendeu disponibilizar uma experiência de realidade aumentada na cidade de Bragança em geral, e no IPB em particular. A ideia central do projeto passou pelo desenvolvimento de uma aplicação que pretendeu levar o utilizador a todos os pontos importantes não só das escolas do IPB mas também da cidade e, quando fisicamente nesses locais, poder aumentar a sua experiência com conteúdos que lhe são apresentados pela aplicação de realidade aumentada. A metodologia de desenvolvimento correspondeu essencialmente na prototipagem rápida da aplicação e na análise e projeto de sistemas numa abordagem iterativa e incremental. Esta aplicação tem duas versões. Uma que funciona com a localização por GPS ativa, apostando na mobilidade através das bicicletas Xispa, existentes em diversos pontos de Bragança e localizadas através do Google maps. A outra que funciona totalmente integrada, sem localização GPS, de modo a que quem esteja distante da cidade de Bragança também possa usufruir desta realidade aumentada. Depois de concluído o projeto, o mesmo foi avaliado através de um estudo de caso que incidiu na observação da sua utilização e no preenchimento de um inquérito por diferentes tipos de discentes e docentes em mobilidade internacional. Salienta-se que a maioria dos inquiridos classificou a aplicação como muito útil e necessária para os alunos e professores em mobilidade. $O$ aspeto apontado como menos interessante foi a necessidade de ser estabelecido o link para a aplicação através do código $Q R$ (QR code) na aplicação com a localização por GPS ativa.

Palavras-chave: Realidade aumentada, inovação formativa, mobilidade, $Q R$ code. 


\section{Introdução}

Muitos artigos se têm escrito sobre realidade virtual, aumentada e mista. Paralelamente, os programas, apps e conteúdos deste tipo têm vindo a ganhar expressão, tanto a nível académico e cultural como a nível comercial e de entretenimento ou de lazer. O projeto "IPB: uma realidade aumentada" pretendeu disponibilizar uma experiência de realidade aumentada (RA) na cidade de Bragança em geral, e no Instituto Politécnico de Bragança (IPB) em particular.

Ao nível de projetos e estudos de RA em Portugal, destaca-se que, no turismo, este tipo de realidade tem contribuído para revolucionar a forma como turistas e visitantes acedem a informação, adquirem conhecimento e se integram nos espaços físicos e destinos visitados (Neto Azevedo \& Alturas, 2019).

Já no contexto educativo, podemos considerar que a inserção de tecnologias na educação tem fomentado novos recursos e experiências educacionais aos seus utilizadores, capazes de auxiliar no processo de ensino e aprendizagem em diferentes áreas. Dentre as tecnologias emergentes, o uso de RA em dispositivos móveis tem-se destacado, em virtude da combinação entre os recursos de multimédia aumentada e de aprendizagem móvel (Herpich, Nunes, Petri, Nicolete, \& Tarouco, 2019).

O projeto que esteve na base deste artigo teve como intuito principal o desenvolvimento de uma aplicação de RA e, previamente, o estudo e projeto da mesma, nomeadamente a identificação e especificação de requisitos da proposta de realidade aumentada a desenvolver e a correspondente escolha e estudo da aplicação de desenvolvimento.

Deste processo resultaram duas versões da aplicação: 1) Uma que funciona com a localização por GPS ativa, apostando na mobilidade através das bicicletas Xispa, existentes em diversos pontos de Bragança e localizadas através do Google maps. 2) A outra que funciona totalmente integrada, sem localização GPS, para que quem esteja distante da cidade de Bragança também possa usufruir desta experiência de RA.

Depois de concluído o projeto, o mesmo foi avaliado através de um estudo de caso que incidiu na observação da sua utilização e no preenchimento de um inquérito por diferentes tipos de discentes e docentes em mobilidade internacional.

Assim, este artigo apresenta, primeiramente, o resultado de uma revisão da literatura sobre o estado da arte da realidade aumentada, consubstanciada pela recolha cronológica e pela identificação das aplicações de desenvolvimento. De seguida, distinguiram-se os conceitos de realidade aumentada, realidade virtual e realidade mista e identificaram-se as aplicações de desenvolvimento gratuitas ou open source para escolher a aplicação de desenvolvimento: 
o Metaverse. Posteriormente, procedeu-se ao planeamento e projeto da visita de realidade aumentada, que orientou o desenvolvimento do aplicativo, que depois de concluído, foi alvo de avaliação através de um estudo de caso.

\section{Metodologías}

O inicio do projeto de realidade aumentada foi dividido em três partes: estudo da cronologia da realidade aumentada; distinção entre realidade aumentada, realidade virtual e realidade mista e, finalmente, escolha de uma aplicação de desenvolvimento de RA gratuita. Posteriormente, procedeu-se à análise e projeto da aplicação, pensando que equipamentos e monumentos da cidade de Bragança seriam incluídos, para além de abranger o IPB e suas escolas. O desenvolvimento da aplicação correspondeu essencialmente à prototipagem rápida da aplicação e à análise e projeto de sistemas (estudo prévio, análise, projeto, programação, instalação, avaliação e manutenção), numa abordagem iterativa e incremental.

A avaliação do aplicativo de RA referido correspondeu a um estudo de caso que incidiu na observação da sua utilização e no preenchimento de um inquérito por diferentes tipos de discentes e docentes em mobilidade internacional.

O método de estudo de caso vai além de um tipo de pesquisa qualitativa, usando uma mistura de evidência quantitativa e qualitativa (Yin, 2010). É importante compreender o como e o porquê das problemáticas envolvidas. Para tal, existem vários tipos de estudos de caso o nforme estes sejam ni os ou $m$ ltiplos podem tam $\mathrm{m}$ ser e plorat rios des ritivos e e planat rios sendo que a da um deles tem espe ifi id ades ni as Os estudos de a so e plorat rios s o o prel dio de uma investiga o posterior Os estudos de a so des ritivos representam a des ri o o mpleta de um fen meno inserido no seu contexto. Os estudos de caso explanatórios procuram estabelecer rela es de causa-efeito.

Este estudo pode ser considerado um estudo des ritivo ni o uma ve que incide na observação e avaliação por inquérito da utilização da apli a o de RA "IPB: uma realidade aumentada".

\subsection{Estado da arte da realidade aumentada}

Nas últimas duas décadas, as aplicações de realidade aumentada têm recebido cada vez mais atenção e o seu uso tem incidido em diferentes áreas do conhecimento. Esses recursos têm permitido o desenvolvimento de novas interfaces que estão mudando a forma como as 
organizações apresentam seus conteúdos, aumentando a eficiência e promovendo a inovação (Becker, Cummins, Davis, Freeman, Giesinger, \& Ananthanarayanan, 2017).

A quantidade de artigos interessantes e atuais sobre a realidade aumentada permitiu-nos concluir que, atualmente, o estado-da-arte da realidade aumentada está em constante desenvolvimento.

A tabela (https://ictevangelist.com/the-new-periodic-table-of-ios-apps-for-ar-and-vr) ) e correspondente descrição de cada uma das aplicações foi crucial no momento de escolha da aplicação. Com o objetivo de encontrar uma aplicação gratuita ou mesmo uma open source, foi definido depois de um estudo minucioso, o uso do Metaverse como app de desenvolvimento (https://studio.gometa.io/).

No que diz respeito ao turismo relacionado diretamente com a realidade aumentada, os estudos produzidos a este respeito são ainda limitados, particularmente na perceção da forma como os utilizadores adotam a tecnologia e sobre que uso fazem dela. Por outro lado, ainda mais limitados são os estudos que consideram o papel dos stakeholders no que diz respeito à implementação da tecnologia de RA (Neto Azevedo \& Alturas, 2019).

\subsubsection{Cronologia da realidade aumentada}

Podemos destacar os seguintes episódios históricos destacados por Richard Corps (2017):

- Em 1901, ocorreu a primeira menção a algo com conceito de realidade aumentada. Foi no livro The Master Key: An Electrical Fairy Tale (A Chave Mestra: um Conto de Fadas Elétrico), onde o escritor L. Frank Baum (o mesmo de O Mágico de $\mathrm{Oz}$ ) descreve um tipo de óculos que dava a capacidade de visualizar características da personalidade de outras pessoas a quem o usasse.

- Em 1962, surge a primeira referência ao conceito de RA quando Morton Heilig construiu uma máquina com tecnologia multi-sensorial imersiva (Sensorama).

- Em 1968, Ivan Sutherland desenvolveu o primeiro head mounted display (HMD) a que chamou The Sword of Damocles, que é um dispositivo usado na cabeça, ou parte integrante de um capacete, que possui um display óptico em frente de um (HMD Monocular) ou de cada olho (HMD Binocular).

- Em 1981, Dan Reitan mapeou as imagens se orientando pelo espaço e utilizou para transmitir ao vivo na televisão.

- Em 1992, apesar do conceito já existir há imenso tempo, o termo Realidade Aumentada foi criado pelo investigador Tom Caudell da Boeing.

- Em 1996, é criado o Cybercode, tecnologia que permitia a criação de imagens estáticas de Realidade Aumentada usando 2D (início do QRCode). 
- Em 1999, a NASA utiliza RA como parte do sistema de navegação dos testes de lançamento da Nave X-38. No mesmo ano, é criado o Eyetap, por Steve Mann.

- Em 2000, é lançado o primeiro jogo portátil de RA chamado de ARQUAKE. No mesmo ano, é criado o primeiro software de RA, por Hirokazu Kato, chamado de ARToolKit.

- Em 2008, a RA é utilizada pela primeira vez na publicidade pela BMW e Esquire.

- Em 2009, nasce o FLARToolKit para que fosse possível trabalhar com o conteúdo da RA em web browsers.

- Em 2016, é lançado o jogo Pokémon Go (o caso de maior sucesso da Realidade Aumentada e seu auge), jogo que teve mais de 1 bilhão de downloads por todo o mundo.

- Em 2017, Apple e Android lançam as versões de RA: Apple ARKIT e Google ARCORE.

\subsubsection{Realidade virtual vs Realidade aumentada vs Realidade mista}

Apesar de os nomes serem próximos, as funções de cada tipologia são distintas. Para exemplificar a explicação, observe a imagem a seguir que exemplifica a distinção entre Realidade virtual (RV) vs Realidade aumentada (RA) vs Realidade mista (RM).

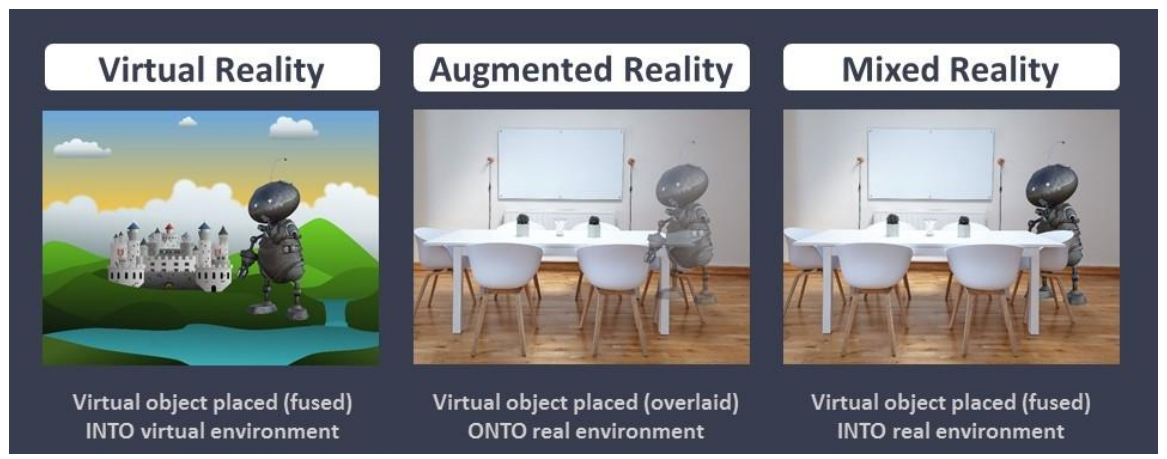

Fig. 1 RV $\times$ RA $\times R M$

Font: http://patriciahaueiss.com/8-major-challenges-the-australian-vr-industry-is-facing/

Basicamente, quando se fala Virtual Reality ou realidade virtual (RV) um "novo mundo" criado e um objeto virtual é colocado para interação com o mesmo, além do que, para ter a imersão completa desta realidade é necessário usar óculos de realidade virtual. Esta é a principal diferença com a Augmented Reality ou realidade aumentada (RA), a qual consiste em apenas sobrepor um objeto virtual ao mundo real, sem ter interação nenhuma com o cenário, e neste caso, apenas um telemóvel com câmera pode ser suficiente para ter a experiência. De salientar que a interação com o objeto virtual não depende da nossa própria localização, já que esse objeto estará sempre à frente da realidade física. Já no caso da Mixed Reality ou realidade mista (RM), como o próprio nome indica, é uma mistura das 
duas realidades, consistindo em um objeto virtual tendo interação com o mundo real, ou seja, a interação com o objeto virtual depende da nossa própria localização.

\subsection{Planeamento e seleção dos locais}

Esta fase correspondeu à pesquisa sobre quais os locais mais adequados e interessantes para a visita, tendo como foco o IPB e os principais monumentos e equipamentos da cidade de Bragança.

Analisados diversos cenários e circuitos turísticos possíveis, foram identificados os locais, devidamente separados por região, nomeadamente: o Castelo de Bragança, sua torre de menagem, o Museu Militar, a Domus, a igreja de Santa Maria (padroeira da cidade) e o Museu Ibérico da Máscara e do Traje. Em outra zona, temos o centro histórico como foco, apresentando o Museu de Arte Contemporânea Graça Morais, o Centro de Fotografia Georges Dussaud, a igreja da Antiga Sé e o Museu do Abade de Baçal. Outros equipamentos são: o Centro de Ciência Viva de Bragança, o Teatro Municipal e a Catedral de Bragança. Por fim, o circuito inclui IPB e as escolas desta instituição de ensino superior público em Bragança, sendo elas: ESA, ESE, ESTiG, ESACT, ESSa.

No âmbito do planeamento, foi também projetada a inclusão das bicicletas Xispa como um meio de transporte, já que é disponibilizado gratuitamente em alguns pontos da cidade.

\subsection{Desenvolvimento da app}

Após devidamente planeada e projetada, foi iniciado o desenvolvimento da aplicação. $\mathrm{O}$ aplicativo foi desenvolvido de forma simples e intuitiva, com o objetivo de apenas trazer informações e inteirar os usuários de uma forma mais divertida sobre a cidade de Bragança.

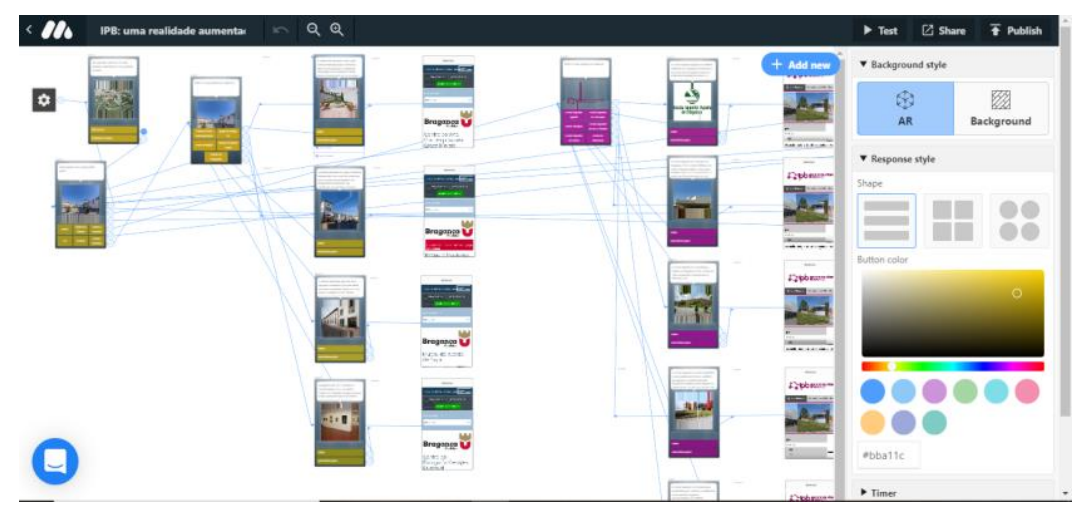

Fig. 2 App em desenvolvimento

Font: Metaverse 
Durante o desenvolvimento foram encontrados alguns obstáculos para que a ideia principal proposta no início fosse colocada em prática, como a junção de todos as miniaplicações, tornando-a uma aplicação completa. Após diversas discussões sobre alternativas para contornar a situação e resolver o problema, acabou por se adiar a solução para a junção da aplicação desenvolvida no Metaverse aos mapas virtuais do trajeto a seguir. A fig. 2 permite ilustrar algumas das cenas que compõem a aplicação de RA, ou seja, mostra as principais cenas e transições no ambiente Metaverse, bem como as hiperligações.

\section{Resultados}

Face aos produtos a disponibilizar, foram encontradas duas propostas de soluções: uma apostou no desenvolvimento da aplicação completa, sem a necessidade de localização, e a outra, um conjunto de miniaplicações, sendo estabelecido o link entre elas através do código QR (QR code) existente em panfletos colocados estrategicamente nos locais de interesse.

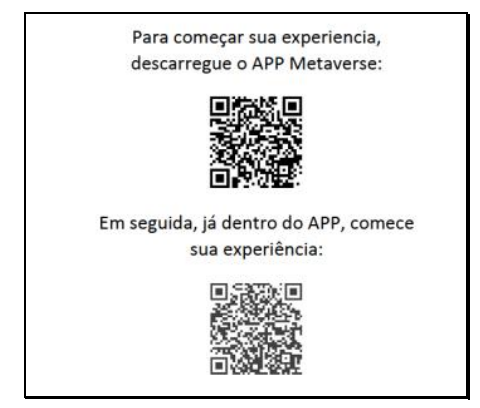

Fig.3 Exemplo de panfleto.

Com estas soluções, o utilizador tinha disponíveis duas opções, uma para visitar Bragança de bicicleta e outra para visitar Bragança sem que estivesse nos locais a visitar.

Para a recolha de dados, foi criado uma sondagem, com a ideia de obter o feedback dos utilizadores, com respostas de avaliação numa escala de Likert de 0 a 5 , em que a opção 0 correspondia à escolha de quem não tinha opinião formada.

O questionário tinha perguntas, tais como: Como avalia a sua experiência com o aplicativo? Quais as chances de recomendar para algum colega? O acesso da aplicação foi fácil? O menu interativo é de fácil manuseio? O que acha que podemos melhorar?.

O questionário estava também disponível em forma de link dentro da aplicação, encontrada na seguinte URL: https://forms.gle/2XLVvuXPMR6etf5k8. 
Estas avaliações permitiram melhorar o aplicativo com o intuito de tornar a experiência de RA mais próxima dos interesses dos utilizadores.

\section{Considerações finais}

Salienta-se que a maioria dos inquiridos classificou a aplicação como muito útil e necessária para os alunos e professores em mobilidade. $\mathrm{O}$ aspeto apontado como menos interessante foi a necessidade de ser estabelecido o link para a aplicação através do código QR (QR code) na aplicação com a localização por GPS ativa.

O desenvolvimento deste estudo de caso permitiu melhorar o aplicativo, procedendo-se à avaliação do funcionamento da app junto de mais utilizadores da comunidade internacional, uma vez que, face à pandemia por COVID-19, esta aplicação poderá ser uma mais valia para mostrar os espaços aos discentes e docentes internacionais. Nesta perspetiva, este projeto foi entretanto reaproveitado pelo Gabinete de Relações Internacionais do IPB no contexto de um estágio curricular que decorreu durante o segundo semestre de 2019/20, tendo sido a base de uma aplicação de RA que foi traduzida para inglês e espanhol e está disponível em https:/emillyselhorst1.wixsite.com/gre-eseb/visita-guiada-virtual-derealidade.

\section{Referências}

Becker, S. A., Cummins, M., Davis, A., Freeman, A., Giesinger, C. H. \& Ananthanarayanan, V. (2017) "NMC Hori o n Report: 2017 Higher Edu ation Edition" Austin: Te as

Byrne, R. (2018). Free Technology for Teachers. Disponível em: https://www.freetech4teachers.com/2018/01/a-periodic-table-of-ar-and-vr-apps.html (Consultado em outubro de 2019)

Gonçalves, V. (2019). ICT in Education. Disponível em: https://www.slideshare.net/VitorGonalves9/tic-na-educao-177686394 (Consultado em novembro de 2019)

Haueiss, P. (2017). 8 major challenges the Australian VR industry is facing right now. Disponível em: $\mathrm{http} / /$ patriciahaueiss.com/8-major-challenges-the-australian-vr-industry-is-facing/ (Consultado em novembro de 2019)

Holland, J. (2019). Augmented Reality: 3D Holograms for Engaged Learning. In X International Conference The Future of Education: Conference Proceedings. Florença. 
Herpich, F., Nunes, F., Petri, G., Nicolete, P. \& Tarouco, L. (2019). Modelo de avaliação de abordagens educacionais em realidade aumentada móvel. RENOTE. Revista Novas Tecnologias na Educação, v. 17, p. 355-364.

Yin, R. K. (2010). Estudo de caso planejamento e métodos (4a Edição ed.). (A. Thorell, Trad.) Porto Alegre: Bookman.

Neto Azevedo, J. \& Alturas, B. (2019) A Realidade Aumentada no Turismo Lisboeta. Proposta de um Modelo de Adoção de Tecnologia de RA. 10.23919/CISTI.2019.8760706. 